\title{
Examples of Surfaces with Canonical Map of Maximal Degree
}

\author{
Ching-Jui Lai* and Sai-Kee Yeung
}

\begin{abstract}
It was shown by Beauville that if the canonical map $\varphi_{\left|K_{M}\right|}$ of a complex smooth projective surface $M$ is generically finite, then $\operatorname{deg}\left(\varphi_{\left|K_{M}\right|}\right) \leq 36$. The first example of a surface with canonical degree 36 was found by the second author. In this article, we show that for any surface which is a degree four Galois étale cover of a fake projective plane $X$ with the largest possible automorphism group $\operatorname{Aut}(X)=C_{7}: C_{3}$ (the unique non-abelian group of order 21), the base locus of the canonical map is finite, and we verify that 35 of these surfaces have maximal canonical degree 36 . We also classify all smooth degree four Galois étale covers of fake projective planes, which give possible candidates for surfaces of canonical degree 36. Finally, we also confirm in this paper the optimal upper bound of the canonical degree of smooth threefolds of general type with sufficiently large geometric genus, related to earlier work of Hacon and Cai.
\end{abstract}

\section{Introduction}

Let $M$ be a smooth complex projective minimal surface of general type with $p_{g}(M) \neq 0$. Assume that the canonical map,

$$
\varphi=\varphi_{\left|K_{M}\right|}: M \rightarrow W:=\overline{\varphi(M)} \subseteq \mathbb{P}^{p_{g}(M)-1}
$$

is generically finite onto its image. We are interested in the canonical degree of $M$, the degree of $\varphi$. If $\varphi$ is not generically finite, we simply say that $M$ has canonical degree zero. The following proposition was proved in [2], cf. [19]. We include the proof here for completeness.

Proposition 1.1. Let $M$ be a minimal surface of general type whose canonical map $\varphi=$ $\varphi_{\left|K_{M}\right|}$ is generically finite. Then $\operatorname{deg} \varphi \leq 36$. Moreover, $\operatorname{deg} \varphi=36$ if and only if $M$ is a smooth ball quotient $\mathbb{B}_{\mathbb{C}}^{2} / \Sigma$ with $p_{g}(M)=3, q(M)=0$, and $\left|K_{M}\right|$ is base point free.

Received September 15, 2020; Accepted January 13, 2021.

Communicated by Jungkai Alfred Chen.

2020 Mathematics Subject Classification. 14J25, 14J29.

Key words and phrases. special surfaces, surfaces of general type, canonical map.

The first author was partially supported by the Ministry of Science and Technology in Taiwan with grant number MOST 107-2115-M-006-020. The second author was partially supported by a grant from the National Science Foundation.

${ }^{*}$ Corresponding author. 
Proof. Let $P$ be the mobile part of $\left|K_{M}\right|$. Let $S \rightarrow M$ be a resolution of $P$ and let $P_{S}$ be the induced base point free linear system defining $S \rightarrow W=\overline{\varphi(M)}$. Then

$$
\operatorname{deg} \varphi \cdot\left(p_{g}-2\right) \leq \operatorname{deg} \varphi \cdot \operatorname{deg} W=P_{S}^{2} \leq P^{2} \leq K_{M}^{2} \leq 9 \chi\left(\mathcal{O}_{M}\right) \leq 9\left(1+p_{g}\right) .
$$

The first inequality is the degree bound for a non-degenerate surface in $\mathbb{P}^{n}$ given in [2], while the fourth inequality is the Bogomolov-Miyaoka-Yau inequality. Hence as $p_{g} \geq 3$, we have

$$
\operatorname{deg} \varphi \leq 9\left(\frac{1+p_{g}}{p_{g}-2}\right) \leq 36 .
$$

Moreover, $\operatorname{deg} \varphi=36$ only when $p_{g}(M)=3, q(M)=0$, and $P_{S}^{2}=P^{2}=K_{M}^{2}$. This is only possible when $\left|K_{M}\right|$ is base point free. In such a case, $K_{M}^{2}=36=9 \chi\left(\mathcal{O}_{M}\right)$ and hence $M$ is a smooth ball quotient $\mathbb{B}_{\mathbb{C}}^{2} / \Sigma$ by results of Aubin and Yau, cf. [1,2].

Notation. Throughout this paper, we do not distinguish line bundles with divisors. The linear equivalence and numerical equivalence of divisors are written respectively as $D_{1} \sim D_{2}$ and $D_{1} \equiv D_{2}$. The cyclic group of order $n$ is denoted by $C_{n}$. The group $C_{7}: C_{3}$ is the unique non-abelian group of order 21. The projective space of dimension $n$ over $\mathbb{C}$ is denoted by $\mathbb{P}^{n}$. A finite field of order $n$ is denoted by $F_{n}$.

From Proposition 1.1, it is an interesting problem to know the geometric realization of possible canonical degrees and many surfaces with canonical degree at most 16 have been constructed, see 9, 15 for more references. However, the first example of a surface with maximal canonical degree 36 was constructed only recently by [19 as a suitably chosen $C_{2} \times C_{2}$-Galois cover of a special fake projective plane $X$. The fake projective plane $X$ in [19 has $\operatorname{Aut}(X)=C_{7}: C_{3}$, and by 14 it satisfies $h^{0}\left(X, 2 L_{X}\right)=0$ for every ample generator $L_{X}$ of $\mathrm{NS}(X)$. The choice of the lattice for the ball quotient $M$ is explicitly described in [19] via the classifying data of [6, 16].

Here are the main goals of this paper. The first goal is to construct more examples of surfaces with maximal canonical degree. This is given as Theorem 1.4 below. Then we examine the corresponding question in complex dimension 3, given as Corollary 1.5 below. The second goal is to identify all potential examples of surfaces of canonical degree 36 constructed as a degree four Galois étale cover of a fake projective plane. We prove that for these Galois covers the canonical maps have at worst discrete base locus whenever the underlying fake projective plane has the largest possible automorphism group $C_{7}: C_{3}$. This is given as Theorem 1.2 and Proposition 1.3 . For the presentation of this paper, we start with Theorem 1.2 hoping that it would give the reader a more comprehensible overall picture.

We remark that our proof of Theorem 1.4 is essentially independent of Theorem 1.2 and Proposition 1.3 A reader who is interested only in new surfaces of canonical degree 36 
may briefly go over statements in earlier sections and proceed directly to Section 4 of the paper.

Recall that a fake projective plane is a ball quotient $X=\mathbb{B}_{\mathbb{C}}^{2} / \Pi$ for some lattice $\Pi \subseteq \mathrm{PU}(2,1)$, where $\Pi$ is constructed as a subgroup of a maximal arithmetic lattice $\bar{\Gamma}$. An unramified cover $M$ of $X$ is given by $\mathbb{B}_{\mathbb{C}}^{2} / \Sigma$ for a normal subgroup $\Sigma \triangleleft \Pi$ of finite index. For the sequence of Galois covers

$$
M:=\mathbb{B}_{\mathbb{C}}^{2} / \Sigma \stackrel{p}{\rightarrow} X=\mathbb{B}_{\mathbb{C}}^{2} / \Pi \stackrel{q}{\rightarrow} \mathbb{B}_{\mathbb{C}}^{2} / \bar{\Gamma}
$$

corresponding to the normal subgroups $\Sigma \triangleleft \Pi \triangleleft \bar{\Gamma}$, one has the covering group $\operatorname{Gal}(M / X)=$ $\Pi / \Sigma$ and $\operatorname{Aut}(X)=\bar{\Gamma} / \Pi$. We focus on the case when $|\operatorname{Gal}(M / X)|=4$ and $\operatorname{Aut}(X)=$ $C_{7}: C_{3}$. Our first theorem identifies potential examples of surfaces of canonical degree 36 .

Theorem 1.2. Let $M \rightarrow X$ be a degree four Galois étale cover over a fake projective plane $X$ with $\operatorname{Aut}(X)=C_{7}: C_{3}$. Then $q(M)=0$ and the base locus of the linear system $\left|K_{M}\right|$ is discrete.

A degree four Galois étale cover $M \rightarrow X$ over a fake projective plane $X$ is determined by a quotient of $H_{1}(X, \mathbb{Z})$ of order four, to be explained in details in Lemma 2.1. The degree of this cover is dictated by the possible existence of a surface of maximal canonical degree, i.e., $K_{M}^{2} / K_{X}^{2}=4$. There are many degree four covers of fake projective planes. For future reference, we classify all such surfaces. In the table below, only lattices of fake projective planes giving rise to Galois étale covers of degree four are listed, which is the case if there is a normal subgroup of index four in the lattice $\Pi$ corresponding to a given fake projective plane $X=\mathbb{B}_{\mathbb{C}}^{2} / \Pi$. This list of the fake projective planes follows the conventions in 6, 16. In the following table, we have

(1) column 1: $k$ is a totally real number field, $\ell$ is a totally imaginary extension of $k$, and $\mathcal{T}$ represents a finite number of places relevant to the classification. These are notations used to classify fake projective planes defined in [16;

(2) column 2: the naming of classes of maximal arithmetic lattices containing fake projective planes in [6] corresponds to $\bar{\Gamma}$ in the notation of [16], where $a$ and $p$ are data from the first column;

(3) column 3: the naming of the individual fake projective planes in each class used in 6 ;

(4) column 4: $\operatorname{Aut}(X)$ is the automorphism group of a fake projective plane $X$;

(5) column 5: the first homology class of a fake projective plane $X$; 
(6) column 6: $N_{0}$ is the number of degree four coverings of $X$, which is the number of subgroups of index four of the lattice $\Pi$;

(7) column 7: $N_{1}$ denotes the number of normal coverings among the degree four coverings above.

All the examples in the last column satisfy $H_{1}(M, \mathbb{Q})=0$, which implies $q(M)=0$ by Poincaré Duality.

\begin{tabular}{|c|c|c|c|c|c|c|}
\hline$(k, \ell, \mathcal{T})$ & class & $X$ & $\operatorname{Aut}(X)$ & $H_{1}(X, \mathbb{Z})$ & $N_{0}$ & $N_{1}$ \\
\hline \multirow{2}{*}{$(\mathbb{Q}, \mathbb{Q}(\sqrt{-1}),\{5\})$} & $(a=1, p=5, \emptyset)$ & $\left(a=1, p=5, \emptyset, D_{3}\right)$ & $C_{3}$ & $C_{2} \times C_{4} \times C_{31}$ & 4 & 3 \\
\hline & $(a=1, p=5,\{2\})$ & $\left(a=1, p=5,\{2\}, D_{3}\right)$ & $C_{3}$ & $C_{4} \times C_{31}$ & 4 & 1 \\
\hline$(\mathbb{Q}, \mathbb{Q}(\sqrt{-1}),\{2,5\})$ & $(a=1, p=5,\{2 I\})$ & $(a=1, p=5,\{2 I\})$ & $\{1\}$ & $C_{2} \times C_{3} \times C_{4}^{2}$ & 47 & 19 \\
\hline \multirow{2}{*}{$(\mathbb{Q}, \mathbb{Q}(\sqrt{-2}),\{3\})$} & $(a=2, p=3, \emptyset)$ & $\left(a=2, p=3, \emptyset, D_{3}\right)$ & $C_{3}$ & $C_{2}^{2} \times C_{13}$ & 4 & 1 \\
\hline & $(a=2, p=3,\{2\})$ & $\left(a=2, p=3,\{2\}, D_{3}\right)$ & $C_{3}$ & $C_{2}^{2} \times C_{13}$ & 4 & 1 \\
\hline$(\mathbb{Q}, \mathbb{Q}(\sqrt{-2}),\{2,3\})$ & $(a=2, p=3,\{2 I\})$ & $(a=2, p=3,\{2 I\})$ & $\{1\}$ & $C_{2}^{4} \times C_{3}$ & 83 & 35 \\
\hline \multirow{5}{*}{$(\mathbb{Q}, \mathbb{Q}(\sqrt{-7}),\{2\})$} & \multirow{2}{*}{$(a=7, p=2, \emptyset)$} & $\left(a=7, p=2, \emptyset, D_{3} 2_{7}\right)$ & $C_{7}: C_{3}$ & $C_{2}^{4}$ & 91 & 35 \\
\hline & & $\left(a=7, p=2, \emptyset, 7_{21}\right)$ & $\{1\}$ & $C_{2}^{2} \times C_{3} \times C_{7}$ & 3 & 1 \\
\hline & \multirow{3}{*}{$(a=7, p=2,\{7\})$} & $\left(a=7, p=2,\{7\}, D_{3} 2_{7}\right)$ & $C_{7}: C_{3}$ & $C_{2}^{3}$ & 7 & 7 \\
\hline & & $\left(a=7, p=2,\{7\}, D_{3} 7_{7}^{\prime}\right)$ & $C_{3}$ & $C_{2}^{2} \times C_{7}$ & 2 & 1 \\
\hline & & $\left(a=7, p=2,\{7\}, 7_{21}\right)$ & $\{1\}$ & $C_{2}^{3} \times C_{3}$ & 19 & 7 \\
\hline \multirow{4}{*}{$(\mathbb{Q}, \mathbb{Q}(\sqrt{-7}),\{2,3\})$} & \multirow{2}{*}{$(a=7, p=2,\{3\})$} & $\left(a=7, p=2,\{3\}, D_{3}\right)$ & $C_{3}$ & $C_{2} \times C_{4} \times C_{7}$ & 4 & 3 \\
\hline & & $\left(a=7, p=2,\{3\}, 3_{3}\right)$ & $\{1\}$ & $C_{2}^{2} \times C_{3} \times C_{4}$ & 19 & 11 \\
\hline & \multirow{2}{*}{$(a=7, p=2,\{3,7\})$} & $\left(a=7, p=2,\{3,7\}, D_{3}\right)$ & $C_{3}$ & $C_{4} \times C_{7}$ & 2 & 1 \\
\hline & & $\left(a=7, p=2,\{3,7\}, 3_{3}\right)$ & $\{1\}$ & $C_{2} \times C_{3} \times C_{4}$ & 7 & 3 \\
\hline$(\mathbb{Q}, \mathbb{Q}(\sqrt{-7}),\{2,5\})$ & $(a=7, p=2,\{5\})$ & $(a=7, p=2,\{5\})$ & $\{1\}$ & $C_{2}^{2} \times C_{9}$ & 3 & 1 \\
\hline \multirow{5}{*}{$(\mathbb{Q}, \mathbb{Q}(\sqrt{-15}),\{2\})$} & \multirow{2}{*}{$(a=15, p=2, \emptyset)$} & $\left(a=15, p=2, \emptyset, D_{3}\right)$ & $C_{3}$ & $C_{2}^{2} \times C_{7}$ & 2 & 1 \\
\hline & & $\left(a=15, p=2, \emptyset, 3_{3}\right)$ & $\{1\}$ & $C_{2}^{3} \times C_{9}$ & 11 & 7 \\
\hline & $(a=15, p=2,\{3\})$ & $\left(a=15, p=2,\{3\}, 3_{3}\right)$ & $C_{3}$ & $C_{2}^{3} \times C_{3}$ & 19 & 7 \\
\hline & $(a=15, p=2,\{5\})$ & $\left(a=15, p=2,\{5\}, 3_{3}\right)$ & $\{1\}$ & $C_{2}^{2} \times C_{9}$ & 3 & 1 \\
\hline & $(a=15, p=2,\{3,5\})$ & $\left(a=15, p=2,\{3,5\}, 3_{3}\right)$ & $C_{3}$ & $C_{2}^{2} \times C_{3}$ & 1 & 1 \\
\hline$\left(\mathcal{C}_{18},\left\{v_{3}\right\}\right)$ & $\left(\mathcal{C}_{18}, p=3, \emptyset\right)$ & $\left(\mathcal{C}_{18}, p=3, \emptyset, d_{3} D_{3}\right)$ & $C_{3} \times C_{3}$ & $C_{2}^{2} \times C_{13}$ & 1 & 1 \\
\hline \multirow{5}{*}{$\left(\mathcal{C}_{20},\left\{v_{2}\right\}\right)$} & $\left(\mathcal{C}_{20},\left\{v_{2}\right\}, \emptyset\right)$ & $\left(\mathcal{C}_{20},\left\{v_{2}\right\}, \emptyset, D_{3} 2_{7}\right)$ & $C_{7}: C_{3}$ & $C_{2}^{6}$ & 651 & 651 \\
\hline & \multirow{2}{*}{$\left(\mathcal{C}_{20},\left\{v_{2}\right\},\{3+\}\right)$} & $\left(\mathcal{C}_{20},\left\{v_{2}\right\},\{3+\}, D_{3}\right)$ & $C_{3}$ & $C_{4} \times C_{7}$ & 2 & 1 \\
\hline & & $\left(\mathcal{C}_{20},\left\{v_{2}\right\},\{3+\},\{3+\}_{3}\right)$ & $\{1\}$ & $C_{2} \times C_{3} \times C_{4}$ & 7 & 3 \\
\hline & \multirow{2}{*}{$\left(\mathcal{C}_{20},\left\{v_{2}\right\},\{3-\}\right)$} & $\left(\mathcal{C}_{20},\left\{v_{2}\right\},\{3-\}, D_{3}\right)$ & $C_{3}$ & $C_{4} \times C_{7}$ & 2 & 1 \\
\hline & & $\left(\mathcal{C}_{20},\left\{v_{2}\right\},\{3-\},\{3-\}_{3}\right)$ & $\{1\}$ & $C_{2} \times C_{3} \times C_{4}$ & 7 & 3 \\
\hline
\end{tabular}

Table 1.1

Proposition 1.3. There are altogether 835 lattices which give rise to 1670 non-biholomorphic smooth minimal surfaces as degree four Galois étale covers of fake projective planes with $q(M)=0$. 
From Table 1.1, there are $35 C_{2} \times C_{2}$ Galois étale covers of the fake projective plane ( $a=7, p=2, \emptyset, D_{3} 2_{7}$ ), which was first constructed as a normal subgroup of index 4 in the maximal arithmetic lattice of its class in [16]. Generalizing the result of [19], we show that these étale covers all have canonical degree 36.

Theorem 1.4. The 35 degree four Galois étale covers of the fake projective plane ( $a=$ $\left.7, p=2, \emptyset, D_{3} 2_{7}\right)$, all with Galois group $C_{2} \times C_{2}$, are minimal surfaces of general type with canonical degree 36 .

Our result has the implication on the optimal canonical degree for smooth threefolds of general type with large geometric genus. We refer the readers to Section 5 for more details.

Corollary 1.5. There exist many examples of smooth minimal threefolds of general type $Y$ with the degree of the canonical map $\operatorname{deg}\left(\Phi_{\left|K_{Y}\right|}\right)=72$. In fact, there exist such threefolds with $p_{g}(Y)=3 g$ and $K_{Y}^{3}=72(g-1)$ for each $g \geq 2$.

The surface studied in [19 has Picard number one, which is a deep result in automorphic forms from [3, 18], and is used in [19] to simplify the geometric arguments. For a general degree four étale cover of a fake projective plane, it is not clear whether the Picard number equals to one. Comparing to the result in [19], one technical improvement in the present article is to show that any surface as in Theorem 1.2 possesses a generically finite canonical map. Continuing from this, mobility of the canonical system is proved but in a different argument from [19]. In fact, we can show that any degree four étale cover of a fake projective plane with $\operatorname{Aut}(X)=C_{7}: C_{3}$ has generically finite canonical map and at worst discrete base locus. To get rid of the finite number of base points, we need more detailed information about the canonical sections as given in [19], see in particular the corrigendum there. By analyzing carefully the method used in [19], we come up with new examples of surfaces with maximal canonical degree by considering new degree four Galois étale covers of the same fake projective plane $X$ used in [19]. These new étale covers correspond to various $C_{2} \times C_{2}$ quotient groups of $H_{1}(X, \mathbb{Z})=C_{2}^{4}$. In such cases, we are able to write down relevant global sections explicitly with the help of Magma and finish the prove of base point freeness. This last step is where we have to restrict further the type of lattice $\Sigma$ associated to $M$.

To find which étale cover works for our scheme, as a first step we list all normal subgroups of index four in a lattice associated to a fake projective plane. All fake projective planes supporting such a subgroup are listed in the third column of Table 1.1 above. Now for each of the listed surfaces, we exhaust all possible normal subgroups of index four. The procedure of finding such a surface as well as verification of necessary conditions stated in Theorem 1.2 and Proposition 1.3 is similar to that in [19]. In [19], the choice of the $C_{2} \times C_{2}$ 
Galois étale cover is very specific and has to come from killing the 2-torsion invariant line bundles under a Sylow 3-subgroup of the automorphism group $C_{7}: C_{3}$. In this paper, we obtain more examples by overcoming this technical hurdle, namely, we consider all possible $C_{2} \times C_{2}$ Galois étale covers of the fake projective plane in [19.

The explicit computation is accomplished by using Magma. The proof of Theorem 1.4 generalizes the argument of [19], see also the Corrigendum in [19] and [17].

Here is the organization of this paper. We first prepare some preliminary results related to our construction in Section 2. The proofs of Theorems 1.2 and 1.4 are given in Sections 3 and 4 respectively. Finally we study the corresponding problem in dimension three in Section 5 .

2. Preliminary discussions and idea of proofs

Let $X=\mathbb{B}_{\mathbb{C}} / \Pi$ be a fake projective plane with $\pi_{1}(X)=\Pi$. It is known from definition that the first Betti number of $X$ is trivial. According to [16, there is always a nontrivial torsion element in $H_{1}(X, \mathbb{Z})$. The torsion group $H_{1}(X, \mathbb{Z})$ is available from $[6]$.

Lemma 2.1. A fake projective plane $X$ possesses a degree four Galois étale cover if and only if there is a quotient group of order four of $H_{1}(X, \mathbb{Z})$.

Proof. We know that $H_{1}(X, \mathbb{Z})$ is a direct sum of finite cyclic abelian groups as the first Betti number of $X$ is trivial. If $Q$ is a quotient group of order four of $H_{1}(X, \mathbb{Z})$, then there is a homomorphism

$$
\rho: \Pi \rightarrow \Pi /[\Pi, \Pi]=H_{1}(X, \mathbb{Z}) \rightarrow Q
$$

The kernel of $\rho$ gives rise to a normal subgroup $\Sigma$ of index four in $\Pi$, with $Q$ as the deck transformation group of the covering map $M=\mathbb{B}_{\mathbb{C}}^{2} / \Sigma \rightarrow X=\mathbb{B}_{\mathbb{C}}^{2} / \Pi$.

On the other hand, if there is a normal subgroup $\Sigma$ of index four in $\Pi$, it leads to a homomorphism $\sigma: \Pi \rightarrow \Pi / \Sigma$. As a group of order four is always abelian, $\sigma$ factors through a homomorphism $\Pi /[\Pi, \Pi] \rightarrow \Pi / \Sigma$. We conclude that $\Pi / \Sigma$ lives as a quotient group of order four of $\Pi /[\Pi, \Pi]=H_{1}(X, \mathbb{Z})$.

We consider an étale cover $\pi: M \rightarrow X$ corresponding to a subgroup $\pi_{1}(M) \leq \Pi$ of index four. In particular, the finite group $\mathcal{G}=\Pi / \pi_{1}(M)$ is either $C_{2} \times C_{2}$ or $C_{4}$.

Lemma 2.2. Let $M$ be a smooth projective surface and assume that there is an étale cover $\pi: M \rightarrow X$ of degree four over a fake projective plane $X$. Suppose that $q(M)=0$, then $p_{g}(M)=3$.

Proof. Since $\pi: M \rightarrow X$ is étale and $p_{g}(X)=q(X)=0, \chi\left(\mathcal{O}_{M}\right)=4 \chi\left(\mathcal{O}_{X}\right)=4$. It follows that $p_{g}(M)=3$ if $q(M)=0$. 
Suppose now a surface $M$ is constructed as in Lemma 2.2. We study the canonical map $\varphi=\varphi_{\left|K_{M}\right|}: M \rightarrow \mathbb{P}^{2}$. We will assume that $\pi: M \rightarrow X$ is a Galois cover, i.e., $\Sigma:=\pi_{1}(M) \leq \Pi$ is normal. Note that then $\left|K_{M}\right|$ is invariant under the Galois group $\mathcal{G}:=\operatorname{Gal}(M / X)=\Pi / \pi_{1}(M)$.

Let us relate the canonical sections from Lemma 2.2 to divisors on $X$. It is known from the Universal Coefficient Theorem that torsions in $H_{1}(X, \mathbb{Z})$ give rise to a torsion line bundle on $X$, cf. Lemma 4 of [14]. Denote by $\mathcal{L}_{\chi}$ the invertible sheaf on $X$ corresponding to a torsion line bundle on $X$ given by a character $\chi$. In this case, the trivial character $\mathcal{O}_{X}$ is denoted by $\mathcal{L}_{1}$. The push forward of the structure sheaf of $M$ splits into eigen-sheaves

$$
\pi_{*} \mathcal{O}_{M}=\bigoplus_{\chi: \mathcal{G} \rightarrow \mathbb{C}^{*}} \mathcal{L}_{\chi}
$$

Denote by $\omega_{M}$ the dualizing sheaf of a surface $M$. Then

$$
\pi_{*} \omega_{M}=\bigoplus_{\chi: \mathcal{G} \rightarrow \mathbb{C}^{*}} \omega_{X} \otimes \mathcal{L}_{\chi}
$$

It follows from the degeneration of the Leray spectral sequence that

$$
H^{i}\left(M, \omega_{M}\right)=\bigoplus_{\chi: \mathcal{G} \rightarrow \mathbb{C}^{*}} H^{i}\left(X, \omega_{X} \otimes \mathcal{L}_{\chi}\right)
$$

for all $i$. Hence vanishing of $q(M)$ implies that $H^{1}\left(X, \omega_{X} \otimes \mathcal{L}_{\chi}\right)=0$ for all $\chi: \mathcal{G} \rightarrow \mathbb{C}^{*}$. By Serre Duality, $h^{2}\left(X, \omega_{X} \otimes \mathcal{L}_{\chi}\right)=h^{0}\left(X, \mathcal{L}_{\chi}^{-1}\right)$, which is either 0 or 1 depending on whether $\chi$ is trivial of not. From Riemann-Roch formula and the fact that $X$ is a fake projective plane, it follows that $h^{0}\left(X, \omega_{X} \otimes \mathcal{L}_{\chi}\right)=1$ for each $\chi \neq 1$, which corresponds to three linearly independent sections in Lemma 2.2. Denote by $D_{1}, D_{2}, D_{3}$ the corresponding curves on $X$. It follows that $H^{0}\left(M, K_{M}\right)$ is generated by $\pi^{*}\left(D_{i}\right), i=1,2,3$, noting that $\pi^{*} \mathcal{L}_{\chi} \cong \mathcal{O}_{M}$

Lemma 2.3. Assume that $q(M)=0$ and let $D_{1}, D_{2}, D_{3}$ be divisors obtained as above. Assume that $D_{1} \cap D_{2} \cap D_{3}=\emptyset$. Then $H^{0}\left(M, K_{M}\right)$ is base point free and the canonical degree of $M$ is 36 .

Proof. Let $x$ be a point in the base point set of $\left|K_{M}\right|$. Since $\left|K_{M}\right|$ is invariant under the Galois group $\mathcal{G}, \pi(x) \in D_{1} \cap D_{2} \cap D_{3}$, which is empty. It follows from Proposition 1.1 that the canonical degree of $M$ is 36 .

The last lemma would be utilized in Section 4 to give a proof of Theorem 1.4. The presentation here is a simplification of the original one, thanks to the suggestion of the referee. 


\section{General constraints on base point set}

The goal of this section is to give a proof of Theorem 1.2, which gives constraints on the base point set of $\left|K_{M}\right|$ without knowledge on an explicit description of fake projective plane $X$. Here as $\rho(X)=1$, we always denote by $L_{X}$ an ample generator of $\operatorname{Pic}(X)$. Also recall that for a fake projective plane $X$, we have $p_{g}(X)=q(X)=0$ and $L_{X}^{2}=1$ by definition. We begin with the following simple observations.

Lemma 3.1. Let $X$ be a fake projective plane and let $L_{X}$ be an ample generator of $\operatorname{Pic}(X)$. Then $h^{0}(X, L) \leq 1$ for any line bundle $L \equiv L_{X}$ and $h^{0}\left(X, L^{\prime}\right) \leq 2$ for any line bundle $L^{\prime} \equiv 2 L_{X}$.

Proof. If $L^{\prime \prime}$ is a line bundle with $L^{\prime \prime} \equiv 4 L_{X}$, then by Riemann-Roch formula $h^{0}\left(X, L^{\prime \prime}\right)=$ 3. But if $L \equiv L_{X}$ and $H^{0}(X, L)$ has two linearly independent sections $x$ and $y$, then $\left\{x^{4}, x^{3} y, x^{2} y^{2}, x y^{3}, y^{4}\right\}$ are five linearly independent sections of $H^{0}\left(X, L^{\otimes 4}\right)$, which is absurd. The second statement is proved similarly.

Lemma 3.2. If $C$ is an irreducible and reduced curve on a fake projective plane $X$ with $C \equiv L_{X}$, then $C$ is smooth of genus 3 .

Proof. Given an irreducible and reduced curve $C$, we denote by $C^{\nu}$ the normalization of $C$ and $\nu: C^{\nu} \rightarrow C$ the normalization morphism. The $\mathcal{O}_{C}$ sheaf $\delta:=\nu_{*} \mathcal{O}_{C^{\nu}} / \mathcal{O}_{C}$ is the cokernel of the natural map $\mathcal{O}_{C} \rightarrow \nu_{*} \mathcal{O}_{C^{\nu}}$ and satisfies

$$
g\left(C^{\nu}\right)=p_{a}\left(C^{\nu}\right)=p_{a}(C)-h^{0}(C, \delta) .
$$

We first remark that $g\left(C^{\nu}\right) \geq 2$ as $X$ is hyperbolic. The Ahlfors-Schwarz Lemma applied to the composition map induced by the normalization $\nu^{\prime}: C^{\nu} \stackrel{\nu}{\rightarrow} C \hookrightarrow X$ (cf. [8]) for the manifolds equipped with Poincaré metrics implies that the Kähler forms satisfy $\nu^{\prime *} \omega_{X} \leq \omega_{C^{\nu}}$, with equality if and only if it is a holomorphic isometry leading to totally geodesic $C$. Since there is no totally geodesic curve on a fake projective plane from the proof of [14, Lemma 6], the inequality is strict. Hence for $C \equiv k L_{X}$ with $k \geq 1$, integrating over $C^{\nu}$, we get

$$
2 k=\frac{2}{3}\left(K_{X} \cdot C\right)<\operatorname{deg}\left(K_{C^{\nu}}\right)=2 g\left(C^{\nu}\right)-2=k(k+3)-2 h^{0}(C, \delta),
$$

where we used the fact that the Ricci curvature is $\frac{3}{2}$ of the holomorphic sectional curvature for the Poincaré metric on $X$ and the adjunction $p_{a}(C)=\frac{1}{2} C \cdot\left(K_{X}+C\right)$. Hence $k=1$ implies that $h^{0}(C, \delta)=0$ and $C$ is smooth with $g(C)=3$.

Lemma 3.3. Let $X$ be a fake projective plane with a nontrivial automorphism group and let $C$ be an effective divisor such that $C \equiv L_{X}$. For any nontrivial subgroup $H \leq \operatorname{Aut}(X)$ with $H \cong C_{3}$ or $C_{7}, h^{*} C \neq C$ for any $h \in H-\{e\}$. 
Proof. Clearly $C$ must be reduced and irreducible as $\rho(X)=1$. From Lemma 3.2, $C$ is smooth of genus three. Suppose now $h^{*} C=C$ for all $h \in H$. From [14, Lemma 6], $H$ must act non-trivially on $C$. Note that $H$ can only be $C_{3}$ or $C_{7}$ from the list of [6].

If $H \cong C_{7}$, then there exists an $H$-fixed point on $C$, as by the Hurwitz formula there is no étale cover of degree 7 from a smooth genus three curve. By [14, Lemma 7], for $x=\operatorname{dim}_{\mathbb{C}} H^{1}\left(C, \mathcal{O}_{C}\right)^{\text {inv }}$, we have the equation

$$
n=2-2 \cdot 3+\frac{2 \cdot 7}{7-1}(3-x) \quad \Longrightarrow \quad 3 n+7 x=9
$$

The only solution is $(n, x)=(3,0)$ and $C / C_{7} \subseteq X / C_{7}$ is a smooth rational curve. But then there is a non-constant lifted map from $\mathbb{P}^{1}$ to the universal cover $\mathbb{B}_{\mathbb{C}}^{2}$ of $X / C_{7}$, this contradicts to Liouville's theorem.

If $H \cong C_{3}$, then there exists an $H$-fixed point on $C$, as by the Hurwitz formula there is no étale cover of degree 3 from a smooth genus three curve. By the same argument as above, we see that $(n, x)=(5,0)$ or $(2,1)$. In either cases, there is a non-constant lifted map from $\mathbb{P}^{1}$ or $\mathbb{C}$ to $\mathbb{B}_{\mathbb{C}}^{2}$, which again contradicts Liouville's theorem.

Lemma 3.4. Let $X$ be a fake projective plane with $\operatorname{Aut}(X)=C_{7}: C_{3}$. Suppose that there is a Galois étale cover $\pi: M \rightarrow X$ of degree four and $q(M)=0$, then the canonical map $\varphi: M \rightarrow \mathbb{P}^{2}$ is generically finite.

Proof. From Lemma 2.2, we know that $p_{g}(M)=3$ and hence the canonical map maps $M$ to $\mathbb{P}^{2}$. Write $\left|K_{M}\right|=P+F$, where $P$ is the mobile part and $F$ is the fixed divisor. By construction, we have $\varphi=\varphi_{\left|K_{M}\right|}=\varphi_{P}: M \rightarrow \mathbb{P}^{2}$. We will abuse the notation: $P$ will be the mobile linear system or a general member in it.

Assume that $\overline{\varphi(M)}=C \subseteq \mathbb{P}^{2}$ is a curve. We will derive a contradiction.

First of all, we claim that $P$ is not base point free, or equivalently $P^{2} \neq 0$. Assume now $P^{2}=0$. We consider $\mathcal{G}=\operatorname{Gal}(M / X)$. Since $g^{*} K_{M}=K_{M}$ for any $g \in \mathcal{G}$, we have that $g^{*} F=F$ for each $g \in \mathcal{G}$. Indeed, $g^{*} P$ is a mobile sub-linear system of $\left|K_{M}\right|$ and hence $g^{*} F \geq F$ as Weil divisors. Hence as $\pi$ is Galois, $F=\pi^{*} F_{X}$ for an effective divisor $F_{X}$ on $X$. Moreover, if $\operatorname{NS}(X)=\left\langle L_{X}\right\rangle$ for an ample divisor $L_{X}$, then $K_{X} \equiv 3 L_{X}, F_{X} \equiv l L_{X}$ for some $0 \leq l \leq 3$, and $P \equiv \pi^{*}(3-l) L_{X}$. Now, $P^{2}=0$ implies that $l=3$ and hence $P \equiv 0$. This is a contradiction as a non-zero effective divisor cannot be numerically trivial.

Since $\varphi: M \rightarrow C \subseteq \mathbb{P}^{2}$ is not a morphism, we take a composition of finitely many smooth blow-ups $\rho: \widehat{M} \rightarrow M$ to resolve $P$ and let $\psi: \widehat{M} \rightarrow C \subseteq \mathbb{P}^{2}$ be the induced morphism. We have the following diagram after taking the Stein factorization of $\psi: S \rightarrow$ 
$C$ :

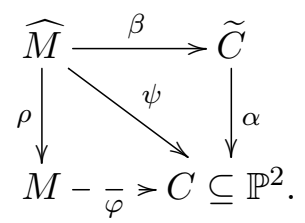

If $\rho^{*} P=\widehat{P}+\widehat{F}$, where $\widehat{P}=\psi^{*}\left|\mathcal{O}_{C}(1)\right|$ is base point free, $\widehat{F} \geq 0$ is the fixed divisor, and $\psi=\psi_{\widehat{P}}$, then $\widehat{F}$ is a non-trivial effective $\rho$-exceptional divisor with $\beta(\widehat{F})=\widetilde{C}$. In particular, $\widetilde{C} \cong \mathbb{P}^{1}$ as all the irreducible components of $\widehat{F}$ are rational. Since $\alpha: \widetilde{C} \rightarrow C$ is defined by $\alpha^{*}\left|\mathcal{O}_{C}(1)\right| \subseteq\left|\mathcal{O}_{\mathbb{P}^{1}}(d)\right|$ for some $d \geq 1$ and hence an element in $\widehat{P}$ is given by $\beta^{*} H$ for some $H \in\left|\mathcal{O}_{\mathbb{P}^{1}}(d)\right|$, we have $\widehat{P} \supseteq \beta^{*}\left|\mathcal{O}_{\mathbb{P}^{1}}(d)\right|$. In particular, we get

$$
\widehat{P}=\psi^{*}\left|\mathcal{O}_{C}(1)\right|=\beta^{*} \alpha^{*}\left|\mathcal{O}_{C}(1)\right|=\beta^{*}\left|\mathcal{O}_{\mathbb{P}^{1}}(d)\right| .
$$

As $\operatorname{dim} \widehat{P}=p_{g}(M)=3$, we get $d=2$ and $C \subseteq \mathbb{P}^{2}$ being irreducible and non-degenerate is a smooth conic in $\mathbb{P}^{2}$.

Let $\widehat{M}_{c}$ be a general fibre of $\widehat{M} \rightarrow \widetilde{C}$ and $D:=\rho_{*}\left(\widehat{M}_{c}\right) \equiv P / 2$ be the corresponding prime divisor on $M$. Recall that $\pi: M \rightarrow X$ is Galois, $K_{M}=\pi^{*} K_{X} \equiv \pi^{*}\left(3 L_{X}\right)$ and $P \equiv \pi^{*}\left(l L_{X}\right)$ for some $1 \leq l \leq 3$ as $P^{2} \neq 0$, where $\operatorname{NS}(X)=\left\langle L_{X}\right\rangle$ and $L_{X}^{2}=1$. It follows from the genus formula,

$$
\left(K_{M}+D\right) \cdot D=2 g_{a}(D)-2 \in 2 \mathbb{Z}
$$

that $l=2$ is the only possibility. Hence $P \equiv \pi^{*}\left(2 L_{X}\right), F=\pi^{*} F_{X} \equiv \pi^{*} L_{X}$, and $D \equiv \pi^{*} L_{X}$. Note that if $h^{0}\left(X, 2 L_{X}\right)=0$ for any ample generator $L_{X}$ on $X$, then we arrive the required contradiction as $2 F_{X} \neq 0$. This is exactly the argument in [19], where the vanishing holds for $X$ a very special fake projective plane as discussed in the introduction. Below we provide a more elementary argument.

It is easy to see that $\mathcal{G}$ acts on $C \cong \mathbb{P}^{1}$ holomorphically and induces an action on $\widetilde{C}$. We claim that there is always a fixed point on $\widetilde{C}=\mathbb{P}^{1}$. If $\mathcal{G}$ acts trivially, then every point is a fixed point 11 Otherwise, $\mathcal{G}$ has two fixed points on $\widetilde{C}$ from the Lefschetz fixed point formula. In particular, the fiber $\widehat{M}_{c}$ over a fixed point $c$ is $\mathcal{G}$-invariant and descends to an effective divisor $G^{X} \equiv L_{X}$ on $X 2$

\footnotetext{
${ }^{1}$ In fact, this case is absurd. If $\mathcal{G}$ acts trivially on $C$, then $\mathcal{G}$ also acts trivially on $\widetilde{C} \cong \mathbb{P}^{1}$. Any fibre of $\beta: \widehat{M} \rightarrow \widetilde{C}$ as a section of $H^{0}\left(\mathbb{P}^{1}, \mathcal{O}_{\mathbb{P}^{1}}(1)\right)$ is $\mathcal{G}$-fixed and descends to a $\mathcal{G}$-invariant section $D \equiv \pi^{*} L_{X}$ on $M$, which then descends to a section $D_{X} \equiv L_{X}$ on $X$. For any two such sections $D$ and $D^{\prime}$ on $M$, $D \sim D^{\prime}$ implies that $D_{X} \equiv D_{X}^{\prime} \equiv L_{X}$ where $\pi^{*} D_{X}=D$ and $\pi^{*}\left(D_{X}^{\prime}\right)=D^{\prime}$. Since $X$ has only finitely many nontrivial torsion but $H^{0}\left(\mathbb{P}^{1}, \mathcal{O}_{\mathbb{P}^{1}}(1)\right)$ is infinite, we can find a line bundle $L=L_{X}+T_{X}$ for some torsion line bundle $T_{X}$ on $X$ with $\operatorname{dim}|L| \geq 1$. This contradicts Lemma 3.1

${ }^{2} \mathrm{Up}$ to here everything works for all fake projective planes with a nontrivial automorphism group.
} 
Suppose now that $\operatorname{Aut}(X)=C_{7}: C_{3}$. Note that in this case a non-trivial torsion elements is always a 2-torsion. In particular for any $\sigma \in \operatorname{Aut}(X), \sigma^{*} G^{X} \sim G^{X}+T_{\sigma}$ for some 2-torsion $T_{\sigma}$ and

$$
\sigma^{*}\left(2 G^{X}\right)=2 \sigma^{*}\left(G^{X}\right) \sim 2 G^{X}+2 T_{\sigma}=2 G^{X}
$$

On the other hand, for any non-trivial element $\sigma \in \operatorname{Aut}(X), G^{X} \neq \sigma^{*} G^{X}$ by Lemma 3.3 . The curves $G^{X}$ and $\sigma^{*} G^{X}$ intersect at a unique point $Q_{\sigma}$ as $G^{X} \cdot\left(\sigma^{*} G^{X}\right)=L_{X}^{2}=1$. We claim that there are three linearly independent sections of the form $2 \sigma^{*} G^{X}$ in $\left|2 G^{X}\right|$, which then contradicts to Lemma 3.1.

We fix one non-trivial $\sigma$ and consider $Q:=Q_{\sigma}$. Note that then $2 G^{X}$ intersects with $\sigma^{*}\left(2 G^{X}\right)$ only at $Q$ with multiplicity four. By the result of [16], the isotropic group at $Q$ cannot be the whole $\operatorname{Aut}(X)$. Hence there exists a nontrivial element $\tau \in \operatorname{Aut}(X)$, $\tau \neq \sigma$, such that $\tau^{*} Q \neq Q$. In particular, $\tau^{*}\left(2 G^{X}\right)$ only intersects with $\tau^{*} \sigma^{*}\left(2 G^{X}\right)$ at $\tau^{*} Q$ with multiplicity four. Since elements in the pencil $\left\langle\mu \cdot 2 G^{X}+\lambda \cdot 2 \sigma^{*} G^{X}\right\rangle$ must pass through $Q$ with multiplicity four, one of $\tau^{*}\left(2 G^{X}\right)$ and $\tau^{*} \sigma^{*}\left(2 G^{X}\right)$ is not in $\left\langle 2 G^{X}, 2 \sigma^{*} G^{X}\right\rangle$ or otherwise $\tau^{*} Q=Q$. Hence $h^{0}\left(X, 2 G^{X}\right)>2$ and we have a contradiction to Lemma 3.1 .

Hence we conclude that $\operatorname{dim} \overline{\varphi(M)} \neq 1$. Since $\varphi(M) \subseteq \mathbb{P}^{2}$ has to be positive dimensional, we conclude that $\varphi: M \rightarrow \mathbb{P}^{2}$ must be dominant and hence generically finite.

Lemma 3.5. Let $M \rightarrow X$ be a Galois étale cover of degree four of a fake projective plane $X$ with $\operatorname{Aut}(X)=C_{7}: C_{3}$. If $q(M)=0$, then the canonical linear system $\left|K_{M}\right|$ is mobile, i.e., there is no codimension one base locus.

Proof. We follow the same notation as in the proof of Lemma 3.4 . NS $(X)=\left\langle L_{X}\right\rangle$ for an ample divisor $L_{X}, K_{X} \equiv 3 L_{X}, F_{X} \equiv l L_{X}$ for some $0 \leq l \leq 3$, and $P \equiv \pi^{*}(3-l) L_{X}$. We claim that $l=0$.

Since $\operatorname{dim} P=p_{g}(M)-1=2>0, P$ contains a nontrivial effective divisor and hence $l \neq 3$.

If $l=1$, then we consider the action of $\operatorname{Aut}(X)=C_{7}: C_{3}$ on $F_{X}=L_{X}+T$, where $T$ is a 2-torsion. Then the same argument as in the proof of Lemma 3.4 produces a line bundle $\mathcal{L} \equiv 2 L_{X}$ with $h^{0}(X, \mathcal{L})>2$, but this violates Lemma 3.1 .

If $l=2$, then we consider the same argument as above on $P_{X} \equiv L_{X}$.

Here is an alternate argument. In the above setting, if $H^{0}\left(X, 2 L_{X}\right)=0$ for $L_{X}$ any ample generator of $\operatorname{Pic}(X)$, then $\left|K_{M}\right|=P$ is mobile. Indeed, the assumption also implies that $H^{0}\left(X, L_{X}\right)=0$ for any ample generator of $\operatorname{Pic}(X)$. Hence for $F=\pi^{*} F_{X}$ with $F_{X} \equiv l L_{X}, l=0$ is the only possibility and $F=0$. The hypothesis holds for any fake projective plane with an automorphism group of order 21 by a result of [14]. 
Proof of Theorem 1.2. First of all, from Magma, all Galois coverings of a fake projective plane of index 4 can be listed, as is done in the proof of Proposition 1.3 below. Furthermore, Magma tells us that abelianization of the lattices associated to such coverings are all trivial. Hence $q(M)=0$ for our examples. Theorem 1.2 now follows from Lemma 3.5 .

Proof of Proposition 1.3. We simply apply the procedure of construction as in [19] to each of the fake projective plane listed in column 3 of Table 1.1. We first need to enumerate all possible surfaces as degree four Galos étale cover associated to fake projective planes as listed. It turns out that the number of index four subgroups of the lattice $\Pi$ to a fake projective plane in the table is recorded in the column $N_{1}$ in Table 1.1. This could be seen by considering subgroups of order 4 in $H_{1}(X, \mathbb{Z})$ as in Lemma 2.1, or by listing index four subgroups of $\Pi$ from Magma.

Now we claim that all the different sub-lattices of index 4 of $\Pi$ in Table 1.1 give rise to non-isometric complex hyperbolic forms in terms of the Killing metrics on the locally symmetric spaces. For this purpose, we assume that $\Lambda_{1}$ and $\Lambda_{2}$ are two groups obtained from the above procedure and $\mathbb{B}_{\mathbb{C}}^{2} / \Lambda_{1}$ is isometric to $\mathbb{B}_{\mathbb{C}}^{2} / \Lambda_{2}$. From construction, $\Lambda_{1}$ and $\Lambda_{2}$ are normal subgroups of index 4 in two lattices $\Pi_{1}$ and $\Pi_{2}$ corresponding to the fundamental groups of fake projective planes. Let $\bar{\Gamma}_{1}$ and $\bar{\Gamma}_{2}$ be the corresponding maximal arithmetic groups in the respective classes. As $\mathbb{B}_{\mathbb{C}}^{2} / \Lambda_{1}$ and $\mathbb{B}_{\mathbb{C}}^{2} / \Lambda_{2}$ are isometric, $\Lambda_{1}$ is conjugate to $\Lambda_{2}$ as discrete subgroups of the same algebraic group $G$ with $G \otimes \mathbb{R} \cong \mathrm{PU}(2,1)$. Hence the two corresponding maximal lattices satisfy $\bar{\Gamma}_{1} \cong \bar{\Gamma}_{2}$, and similarly $\Pi_{1} \cong \Pi_{2}$. It follows that they have to come from the same row in Table 1.1 and hence correspond to the same subgroup of index 4 in the same lattice associated to some fake projective plane. Hence there are altogether 835 non-isometric complex two ball quotients obtained in this way, by summing over the column of $N_{1}$ in Table 1.1 .

Now for each locally symmetric space $M=\mathbb{B}_{\mathbb{C}}^{2} / \Lambda$ obtained as above, it gives rise to a pair of complex structures $J_{1}$ and $J_{2}$, which are conjugate to each other. These two complex structures give rise to two non-biholomorphic complex surfaces $S_{1}=\left(M, J_{1}\right)$ and $S_{2}=\left(M, J_{2}\right)$. In fact, if they are biholomorphic, the corresponding four-fold quotient $S_{1} /[\Pi, \Lambda]$ and $S_{2} /[\Pi, \Lambda]$ are biholomorphic and are fake projective space. This contradicts the results in [13], see also the Addendum of [16], that conjugate complex structures on a fake projective space give rise to two different complex structures.

In general, let $\left(M_{1}, J_{1}\right)$ and $\left(M_{2}, J_{2}\right)$ be two complex ball quotients obtained from taking degree 4 étale covers of some possibly different fake projective planes. If $\left(M_{1}, J_{1}\right)$ and $\left(M_{2}, J_{2}\right)$ are biholomorphic, they are isometric with respect to the corresponding Bergman (Killing) metrics. Hence from the earlier argument, $M_{1}$ is isometric to $M_{2}$ and we may regard $M_{1}=M_{2}$. Now the argument of the last paragraph implies that $J_{1}=J_{2}$. In conclusion, we conclude that the 1670 complex surfaces obtained from the pair of 
conjugate complex structures on the 835 underlying locally symmetric structures give rise to distinct complex surfaces. This concludes the proof of Proposition 1.3 .

\section{New examples of surfaces with maximal canonical degree}

Our goal in this section is to prove Theorem 1.4. The surface studied in [19] and here is constructed from the fake projective plane $X$ given in [16, Section 5.9] in the class of $(a=7, p=2)$ and is denoted by $\left(a=7, p=2, \emptyset, D_{3} 2_{7}\right)$ in the notation of 6 .

Proof of Theorem 1.4. We consider $\pi: M \rightarrow X$ a Galois $C_{2} \times C_{2}$-étale cover of the fake projective plane $X$ in the class $\left(a=7, p=2, \emptyset, D_{3} 2_{7}\right)$. From Magma computation, the irregularity $q(M)=0$, cf. Proposition 1.3 . Hence by Lemma 2.3, it suffices for us to prove that the canonical map of $M$ is base point free. From the discussion in Section 2 , there are non-trivial 2-torsions $\tau_{i} \in \operatorname{Pic}^{0}(X)$ for $i=1,2,3$ corresponding to characters of $\mathcal{G}=\operatorname{Gal}(M / X)=C_{2} \times C_{2}$ such that $H^{0}\left(X, K_{X}+\tau_{i}\right)=\left\langle t_{i}\right\rangle$ and $H^{0}\left(M, K_{M}\right)=\left\langle\pi^{*} t_{i}\right| i=$ $1,2,3\rangle$.

For the convenience of the reader, we recall the key steps of the argument in [19]. For simplicity, we denote by $G$ the automorphism group $\operatorname{Aut}(X)=C_{7}: C_{3}$. The automorphism group of $X$ has a presentation $G=\left\langle a, b \mid a^{7}=b^{3}=1, b a b^{-1}=a^{2}\right\rangle$. The group $G$ contains a normal Sylow 7-subgroup $G_{7}=\langle a\rangle$, and seven conjugate Sylow 3subgroups, one of which is $G_{3}:=\langle b\rangle$. We know from the Riemann-Roch formula that $h^{0}\left(X, 2 K_{X}\right)=10$. In terms of the explicit basis of $H^{0}\left(X, 2 K_{X}\right)$ given by [4, the action of $G$ is presented by

$$
\begin{aligned}
& a\left(u_{0}: u_{1}: u_{2}: u_{3}: u_{4}: u_{5}: u_{6}: u_{7}: u_{8}: u_{9}\right) \\
= & \left(u_{0}: \zeta_{7}^{6} u_{1}: \zeta_{7}^{5} u_{2}: \zeta_{7}^{3} u_{3}: \zeta_{7} u_{4}: \zeta_{7}^{2} u_{5}: \zeta_{7}^{4} u_{6}: \zeta_{7} u_{7}: \zeta_{7}^{2} u_{8}: \zeta_{7}^{4} u_{9}\right) \\
& \quad b\left(u_{0}: u_{1}: u_{2}: u_{3}: u_{4}: u_{5}: u_{6}: u_{7}: u_{8}: u_{9}\right) \\
= & \left(u_{0}: u_{2}: u_{3}: u_{1}: u_{5}: u_{6}: u_{4}: u_{8}: u_{9}: u_{7}\right) .
\end{aligned}
$$

From the Corrigendum of 19$]$, under the action of $G_{7}, S:=\bigcup_{\Sigma \in C_{2}^{4}-\{1\}} H^{0}\left(X, K_{X}+\right.$ $\Sigma)$ consists of 3 orbits, where we recall that a $p$-torsion element $\Sigma \in H_{1}(X, \mathbb{Z})=C_{2}^{4}$ correspond to a $p$-torsion element $\Sigma \in \operatorname{Pic}^{0}(X)$ by the universal coefficient theorem (see [14. Lemma 4]).

(i) $\left\langle\widetilde{t}_{0}\right\rangle=H^{0}\left(X, K_{X}+\Sigma_{0}\right)$, where $\Sigma_{0}$ is $G$-invariant corresponding to an element in $H_{1}(X / G, \mathbb{Z})^{\times}$and $\widetilde{t}_{0}^{2}=u_{0}$.

(ii) Two disjoint $G_{7}$ orbits $\langle a\rangle \widetilde{t}_{1}$ and $\langle a\rangle \widetilde{t}_{2}$, where $\widetilde{t}_{i}$ 's are $G_{3}$-invariant corresponding to elements in $H_{1}\left(X / G_{3}, \mathbb{Z}\right)^{\times}-\left\{\Sigma_{0}\right\}$. 
Let $v_{0}=u_{0}, v_{1}=u_{1}+u_{2}+u_{3}, v_{2}=u_{4}+u_{5}+u_{6}$, and $v_{3}=u_{7}+u_{8}+u_{9}$. From [19], one finds that

$$
\left\{\begin{array}{l}
\widetilde{t}_{0}^{2}=v_{0} \\
\widetilde{t}_{1}^{2}=v_{0}+\frac{1}{2}(1+\sqrt{-7}) v_{1} \\
\widetilde{t}_{2}^{2}=v_{0}+(-5+\sqrt{-7}) v_{1}+4(1-\sqrt{-7}) v_{2}-4\left(v_{3}\right)
\end{array}\right.
$$

with the help of elementary command IsDomain in Magma. It is proved that $\bigcap_{i=0}^{2} Z_{t_{i}^{2}}=\emptyset$, which was verified in the Corrigendum of 19 by checking that $\bigcap_{i=0}^{2} Z_{t_{i}^{2}}=\emptyset$ on $X$ modulo $p=23$ from the command HilbertPolynomial in Magma. We remark that the same example was also studied later in [17], where the author independently verified with more sophisticated techniques in Magma that the sections obtained from the above procedure do give rise to sections in $H^{0}\left(M, K_{M}\right)$.

Now under the action of $G_{7}$, the explicit sections $\widetilde{t}_{0}$ and $a^{j} \widetilde{t}_{i}, i=1,2$ and $0 \leq j \leq 6$, precisely give the effective sections of $S:=\bigcup_{\Sigma \in C_{2}^{4}-\{1\}} H^{0}(X, K+\Sigma)$. We will prove that $\bigcap_{i=0}^{2} Z_{t_{i}^{2}}=\emptyset$ by considering possible choices of $\left\{t_{1}, t_{2}, t_{3}\right\} \subseteq S=\left\langle\widetilde{t}_{0}\right\rangle \cup\langle a\rangle \widetilde{t}_{1} \cup\langle a\rangle \widetilde{t}_{2}$ and checking by Magma whether these sections have common intersection.

Conjugating by an element in $G_{7}$, we may assume that $t_{1}$ belongs to $\left\{\widetilde{t}_{0}, \widetilde{t}_{1}, \widetilde{t}_{2}\right\}$. Suppose $t_{1}=\widetilde{t}_{0}$, where $\widetilde{t}_{0}$ is invariant as a set under $G$, then conjugate by an element in $G_{7}$, we may assume that $t_{2}=\widetilde{t}_{1}$. But by construction $\tau_{3}=\tau_{1} \cdot \tau_{2}$ is determined by $\tau_{1}=\sigma_{0}$ and $\tau_{2}$, which gives $\widetilde{t}_{2} \in H^{0}\left(X, K_{X}+\tau_{0} \cdot \tau_{1}\right)=H^{0}\left(X, K_{X}+\tau_{2}\right)$. In particular, this case was already checked in $\left[19\right.$ as $\bigcap_{i=0}^{2} Z_{t_{i}^{2}}=Z_{v_{0}} \cap Z_{\widetilde{t}_{1}^{2}} \cap Z_{\widetilde{t}_{2}^{2}}=\emptyset$ and we are done.

Consider now the case that none of $t_{i}$ 's is $\tilde{t}_{0}$. In this scenario, $t_{i}$ belongs to the orbits of $\widetilde{t}_{1}$ or $\widetilde{t}_{2}$. Again we use the fact that effective divisors $D_{i}$ 's have common intersections if and only if $2 D_{i}$ 's have common intersections. Hence it suffices for us to prove the following claim.

Lemma 4.1. Let $i, j \in\{1, \ldots, 6\}$. Then

(a) $Z_{\widetilde{t}_{1}} \cap Z_{a^{i} \widetilde{t}_{1}} \cap Z_{a^{j} \widetilde{t}_{2}}=\emptyset$ for $1 \leq i, j \leq 6$;

(b) $Z_{\widetilde{t}_{2}} \cap Z_{a^{i} \widetilde{t}_{1}} \cap Z_{a^{j} \widetilde{t}_{2}}=\emptyset$ for $1 \leq i, j \leq 6$;

(c) $Z_{\widetilde{t}_{1}} \cap Z_{a^{i} \widetilde{t}_{1}} \cap Z_{a^{j} \widetilde{t}_{1}}=\emptyset$ for $1 \leq i<j \leq 6$;

(d) $Z_{\widetilde{t}_{2}} \cap Z_{a^{i} \widetilde{t}_{2}} \cap Z_{a^{j} \widetilde{t}_{2}}=\emptyset$ for $1 \leq i<j \leq 6$.

Proof. In terms of the basis chosen with action of $G_{7}$ given in 4.1 and the explicit sections listed in 4.2, statement (a) in Lemma 4.1 holds if there is no the common intersection 
for the following sections,

$$
\begin{aligned}
& \left\{u_{0}+\frac{1}{2}(1+\sqrt{-7})\left(u_{1}+u_{2}+u_{3}\right), u_{0}+\frac{1}{2}(1+\sqrt{-7})\left(\zeta_{7}^{-i} u_{1}+\zeta_{7}^{-2 i} u_{2}+\zeta_{7}^{-4 i} u_{3}\right),\right. \\
& u_{0}+(-5+\sqrt{-7})\left(\zeta_{7}^{-j} u_{1}+\zeta_{7}^{-2 j} u_{2}+\zeta_{7}^{-4 j} u_{3}\right)+4(1-\sqrt{-7})\left(\zeta_{7}^{j} u_{4}+\zeta_{7}^{2 j} u_{5}+\zeta_{7}^{4 j} u_{6}\right) \\
& \left.\quad-4\left(\zeta_{7}^{j} u_{7}+\zeta_{7}^{2 j} u_{8}+\zeta_{7}^{4 j} u_{9}\right)\right\} .
\end{aligned}
$$

Instead of using the command HilbertPolynomial over the cyclotomic field $\mathbb{Q}\left(\zeta_{7}\right)$ on $X$, we specialize it to the finite field $F_{29}$, where 16 is a primitive 7 -th root of unity and 14 serves as $\sqrt{-7}$. In this way, computing over the finite field $F_{29}$, we verify from Magma that the above three polynomials do not have common intersection on $X$ for all $i, j \in\{1, \ldots, 6\}$ in $F_{29}$. This implies that the original equations do not have common zero over the algebraic number field $\mathbb{Q}\left(\zeta_{7}\right)$. Similar arguments applies to $(\mathrm{b}),(\mathrm{c})$, and $(\mathrm{d})$ in Lemma 4.1

We remark that Lemma 4.1 actually is stronger than what is sufficient for our purpose. For example, consider the case of (a). It is enough to check $Z_{\widetilde{t}_{1}} \cap Z_{a^{i} \widetilde{t}_{1}} \cap Z_{a^{j} \widetilde{t}_{2}}=\emptyset$ for one pair of $(i, j)$ corresponding to the elements $\mathcal{G}-\{1\}=\left\{\tau_{1}, \tau_{2}, \tau_{3}\right\}$. However, since we are checking by Magma, the extra computation does not make any essential difference in computer time. Similar argument applies to the cases (b), (c), (d) as well.

Theorem 1.4 follows immediately from Lemma 4.1 .

\section{Remark on maximal canonical degree of threefolds}

Theorem 1.4 has an implication on the canonical degree bound of threefolds. The purpose of this section is to explain literatures in this direction and relations to Theorem 1.4 . From this point on, let $Y$ be a Gorenstein minimal complex projective threefold of general type with locally factorial terminal singularities. Suppose that the linear system $\left|K_{Y}\right|$ defines a generically finite map $\Phi=\Phi_{\left|K_{Y}\right|}: Y \rightarrow \mathbb{P}^{p_{g}(Y)-1}$. Chen asked in $[7]$ if there is an upper bound of $\operatorname{deg}(\Phi)$. A positive answer was provided in $[11]$ with $\operatorname{deg}(\Phi) \leq 576$. Later on, it was improved in $\left[10\right.$ that $\operatorname{deg}(\Phi) \leq 360$ (with equality if and only if $p_{g}(Y)=4, q(Y)=2$, $\chi\left(\omega_{Y}\right)=5, K_{Y}^{3}=360$, and $\left|K_{Y}\right|$ is base point free). In [5], it is shown that $\operatorname{deg}(\Phi) \leq 72$ if the geometric genus satisfies $p_{g}(Y)>10541$.

As a corollary of Theorem 1.4 and the above discussion, we conclude that the canonical degree 72 can be achieved as stated in Corollary 1.5

Proof of Corollary 1.5. Equipped with Theorem 1.4, the corollary follows essentially from an observation of [5, Section 3].

Take $C$ a smooth hyperelliptic curve of genus $g \geq 2$, then the canonical map $\varphi_{\left|K_{C}\right|}: C \rightarrow$ $\mathbb{P}^{g-1}$ is the composition of the double cover $C \rightarrow \mathbb{P}^{1}$ with the $(g-1)$-Veronese embedding 
$\mathbb{P}^{1} \hookrightarrow \mathbb{P}^{g-1}$. In particular, $\operatorname{deg}\left(\varphi_{\left|K_{C}\right|}\right)=2$, cf. 12 . Take $M$ a surface satisfying the optimal degree bound $\operatorname{deg}\left(\varphi_{\left|K_{M}\right|}\right)=36$ as in Theorem 1.4 , then $\varphi=\varphi_{\left|K_{M}\right|}: M \rightarrow \mathbb{P}^{2}$ is a generically finite morphism of $\operatorname{deg}(\varphi)=K_{M}^{2}=36$.

Now let $Y=X \times C$, then $Y$ is a smooth projective threefold of general type with $p_{g}(Y)=3 g$ and $\Phi=\Phi_{\left|K_{Y}\right|}: Y \rightarrow \mathbb{P}^{3 g-1}$ a morphism. From our construction, it follows that $\Phi$ is generically finite and

$$
\operatorname{deg} \Phi \cdot \operatorname{deg} W=K_{Y}^{3}=3 K_{X}^{2} \cdot K_{C}=3 \cdot 36 \cdot(2 g-2)
$$

where $W=\Phi(Y)$ is the image of the composition maps $Y \hookrightarrow \mathbb{P}^{2} \times \mathbb{P}^{g-1} \hookrightarrow \mathbb{P}^{3 g-1}$ defined by $\left|K_{Y}\right|$ and $\mathcal{O}_{\mathbb{P}^{2} \times \mathbb{P}^{g-1}}(1,1)$. Hence $\operatorname{deg} W=3(g-1)$ and $\operatorname{deg}(\Phi)=72$.

\section{Acknowledgments}

It is a pleasure for the second author to thank Donald Cartwright for his help on Magma commands. The authors would like to express their appreciation and thankfulness to the referees for very helpful comments and suggestions on the paper. This work is partially done during the first author's visit at Research Institute of Mathematical Sciences in Kyoto, National Center of Theoretical Sciences and National Taiwan University in Taiwan, and the second author's visit of the Institute of Mathematics of the University of Hong Kong. The authors thank the warm hospitality of the institutes.

\section{References}

[1] W. P. Barth, K. Hulek, C. A. M. Peters and A. Van de Ven, Compact Complex Surfaces, Second edition, Ergebnisse der Mathematik und ihrer Grenzgebiete 3, SpringerVerlag, Berlin, 2004.

[2] A. Beauville, L'application canonique pour les surfaces de type général, Invent. Math. 55 (1979), no. 2, 121-140.

[3] D. Blasius and J. Rogawski, Cohomology of congruence subgroups of $\mathrm{SU}(2,1)^{p}$ and Hodge cycles on some special complex hyperbolic surfaces, in: Regulators in Analysis, Geometry and Number Theory, 1-15, Progr. Math. 171, Birkhäuser Boston, Boston, MA, 2000.

[4] L. A. Borisov and J. Keum, Explicit equations of a fake projective plane, Duke Math. J. 169 (2020), no. 6, 1135-1162.

[5] J.-X. Cai, Degree of the canonical map of a Gorenstein 3-fold of general type, Proc. Amer. Math. Soc. 136 (2008), no. 5, 1565-1574. 
[6] D. I. Cartwright and T. Steger, Enumeration of the 50 fake projective planes, C. R. Math. Acad. Sci. Paris 348 (2010), no. 1-2, 11-13.

[7] M. Chen, Weak boundedness theorems for canonically fibered Gorenstein minimal 3folds, Proc. Amer. Math. Soc. 133 (2005), no. 5, 1291-1298.

[8] Z.-H. Chen, S. Y. Cheng and Q. K. Lu, On the Schwarz lemma for complete Kähler manifolds, Sci. Sinica 22 (1979), no. 11, 1238-1247.

[9] R. Du and Y. Gao, Canonical maps of surfaces defined by abelian covers, Asian J. Math. 18 (2014), no. 2, 219-228.

[10] _ On the canonical degrees of Gorenstein threefolds of general type, Geom. Dedicata 185 (2016), 123-130.

[11] C. D. Hacon, On the degree of the canonical maps of 3-folds, Proc. Japan Acad. Ser. A Math. Sci. 80 (2004), no. 8, 166-167.

[12] R. Hartshorne, Algebraic Geometry, Graduate Texts in Mathematics 52, SpringerVerlag, New York-Heidelberg, 1977.

[13] V. S. Kulikov and V. M. Kharlamov, On real structures on rigid surfaces, Izv. Ross. Akad. Nauk Ser. Mat. 66 (2002), no. 1, 133-152.

[14] C.-J. Lai and S.-K. Yeung, Exceptional collection of objects on some fake projective planes, submitted.

[15] U. Persson, Double coverings and surfaces of general type, in: Algebraic Geometry (Proc. Sympos., Univ. Troms $\varnothing$, Troms $\varnothing$, 1977), 168-195, Lecture Notes in Math. 687, Springer, Berlin, 1978.

[16] G. Prasad and S.-K. Yeung, Fake projective planes, Invent. Math. 168 (2007), no. 2, 321-370. Addendum: Invent. Math. 182 (2010), no. 1, 213-227.

[17] C. Rito, Surfaces with canonical map of maximum degree, to appear in J. Algebraic Geom.

[18] J. D. Rogawski, Automorphic Representations of Unitary Groups in Three Variables, Annals of Mathematics Studies 123, Princeton University Press, Princeton, NJ, 1990.

[19] S.-K. Yeung, A surface of maximal canonical degree, Math. Ann. 368 (2017), no. 3-4, 1171-1189. Corrigendum, http://www.math.purdue.edu/ yeung/papers/cor.pdf, submitted. 
Ching-Jui Lai

Department of Mathematics, National Cheng Kung University, Tainan 70101, Taiwan E-mail address: cjlai72@mail.ncku.edu.tw

Sai-Kee Yeung

Mathematics Department, Purdue University, West Lafayette, IN 47907, USA

E-mail address: yeung@math.purdue.edu 